\title{
Organización matemática en torno a las funciones logarítmica y exponencial
}

Mathematical organization around functions logarithmic and exponential

\author{
Yesenia Carolina Acuña Mazo yesecaroam_16@hotmail.com \\ Código ORCID: 0000-0002-9663-0204 \\ Ministerio del Poder Popular para la Educación, Venezuela \\ Recibido septiembre 2019 | Arbitrado octubre 2019 | Publicado enero 2020
}

\section{Resumen}

Palabras clave: Organización matemática; Organización didáctica; Teoría antropológica de lo didáctico; Función Logarítmica; Función Exponencial

\section{Abstract}

Keywords: Mathematical organization; Didactic organization; Anthropological theory of the didactic; Logarithmic function; Exponential function
La presente investigación tuvo como propósito analizar la práctica pedagógica del profesor de matemática en torno a las funciones logarítmica y exponencial a nivel de cuarto año. La actividad referida se interpreta y describe desde la teoría antropológica de lo didáctico en consecuencia, en términos de las nociones de organización matemática y organización didáctica. El estudio estuvo constituido por un trabajo documental que permitió elaborar una organización matemática de referencia y por un trabajo de campo con diseño etnográfico. Para ello, se utilizó el análisis de contenido, análisis del discurso y la observación no participante. Como unidades de estudio se seleccionaron la organización didáctica y matemática local de dos profesores de esta área de dos instituciones educativas. Los resultados develaron actividades docentes dirigidas a la presentación de elementos tecnológico-teóricos con una técnica correspondiente asociadas a actividades matemáticas inapropiadas e incompletas; centradas en el bloque práctico-técnico con ausencia de cuestionamientos tecnológico-teóricos.

The purpose of this research was to analyze the pedagogical practice of the mathematics teacher around the logarithmic and exponential functions at the fourth year level. The referred activity is interpreted and described from the anthropological theory of the didactic consequently, in terms of the notions of mathematical organization and didactic organization. The study consisted of a documentary work that developed a mathematical reference organization and a field work with ethnographic design. For this, content analysis, discourse analysis and non-participant observation are used. As units of study, the local didactic and mathematical organizations of two teachers from this area of two educational institutions were selected. the results revealed teaching activities aimed at the presentation of technological-theoretical elements with a corresponding technique associated with appropriate and incomplete mathematical activities; focused on the technical-technical block with no technological-theoretical questions. 


\section{INTRODUCCIÓN}

La educación, constituye un derecho $\mathrm{y}$ necesidad esencial que debe permitir al hombre incorporarse e interactuar en su entorno social, dando lugar a su desarrollo y consecuentes transformaciones sociales en la construcción de su bienestar. De hecho, la misma compone un elemento crucial en la formación y desarrollo del ser humano. Así, el docente, en especial el profesor de matemática, como representante de la institución escolar, es formado para desarrollar, en conjunto con su comunidad educativa, el estudio de una actividad matemática, orientada a cumplir requerimientos emanados de importantes instituciones sociales que le rodean.

Al mismo tiempo, el sistema de enseñanza y la actividad de enseñanza, en particular, mediante la cual se realiza el proceso educativo (actividad docente) debe propiciar actividades de aprendizaje que contribuyan tanto a una formación integral como a la construcción de herramientas que faciliten al individuo actuar de manera autónoma, deliberada, consciente y crítica, conforme a las necesidades de la nación y sociedad en la cual se desenvuelve.

Desde este punto de vista, la Organización de las Naciones Unidas para la Educación la Ciencia y la Cultura, en conjunto con el Instituto Internacional para la Educación Superior en América Latina y el Caribe (UNESCO-IESALC, 2010) consideraron que la educación es un derecho humano y un deber esencial para el total desarrollo del potencial humano. Así pues, tanto el desarrollo como la prosperidad económica dependen de la habilidad de los países del mundo para brindar educación a todos los miembros de su sociedad; ofreciendo un aprendizaje significativo a lo largo de la vida. Una educación de calidad, desde la infancia hasta la edad adulta, a través de los sistemas formales y no formales.

En el marco de estos principios generales, el sistema educativo venezolano intentó desarrollar sus políticas educativas; en efecto, la
Ley Orgánica de Educación (2009) en el artículo 4 estableció:

La educación como derecho humano y deber social fundamental orientada al desarrollo del potencial creativo de cada ser humano en condiciones históricamente determinadas, constituye el eje central en la creación, transmisión y reproducción de las diversas manifestaciones y valores culturales, invenciones, expresiones, representaciones $\mathrm{y}$ características propias para apreciar, asumir y transformar la realidad. (p. 2)

En tal sentido, se requiere que la actividad de enseñanza de una disciplina se enmarque, flexiblemente bajo procesos que conduzcan, entre otros aspectos, hacia el desarrollo integral del individuo. En el caso de la matemática, disciplina científica de relevancia educativa altamente reconocida y requerida por la sociedad, se asume que su enseñanza debe encaminarse hacia el logro de las metas educativas señaladas, pero sin desnaturalizarla, es decir, teniendo presente su propia naturaleza.

Dentro de este contexto, la enseñanza de la matemática debe apoyarse en una visión coherente de su actividad, así como en la disponibilidad de recursos humanos, organizativos y materiales que permitan realizar actividades docentes correspondidas, en un mayor grado, con las tareas y retos que presentan el momento actual y futuro próximo a los estudiantes, pero, en una visión sinérgica con la actividad matemática.

En este orden de ideas, la actividad docente en matemática en torno a la noción de función, en especial, las funciones logarítmica y exponencial ha de orientarse hacia el logro de metas ya descritas, en conjunto al desarrollo de actividades matemáticas y didácticas, en acuerdo a exigencias del momento histórico, 
entorno social $\mathrm{y}$ cultural del individuo; subrayando, tanto la pertinencia como el significado real que estos objetos matemáticos poseen en diversas áreas científicas del hacer humano, al facilitar, por ejemplo: la determinación de la magnitud de un sismo, el decaimiento radiactivo, crecimiento poblacional, inversiones económicas, intensidad del sonido entre otros. (Szemruch y Vaccaro, 2002).

Sin embargo, partiendo de una revisión a estudios en torno a la problemática de la actividad docente del profesor de matemática en Latinoamérica, tales como: (Gascón, 2001; Bastán, Buffarini, Lisera y Roso, 2006; Báez, Cantú y Gómez, 2007) entre otros, se evidencia, no solo la gran distancia que separa la actividad docente matemática real de aquella descrita, pero también el reconocimiento que estos profesionales hacen de algunos factores condicionantes de su actividad, tales como su formación académica, sus concepciones y las estrategias apropiadas para la enseñanza de la matemática.

Ellos sugieren al mismo tiempo, la necesidad de efectuar modificaciones en la dimensión instruccional, curricular e institucional de su práctica docente efectiva. Por otra parte, el informe del Banco Interamericano de Desarrollo (BID) presentado en el año 2010 sobre la Condición de la Educación Matemáticas y Ciencias Naturales en América Latina y el Caribe (ALC), apunta un bajo rendimiento de los estudiantes; lo que trae consigo, existencia de una baja calidad de instrucción, lo cual, a su vez, limita las alternativas orientadas al logro del aprendizaje de las destrezas requeridas en matemática y ciencias naturales, pese a que los profesores del continente, en su mayoría, poseen el nivel de formación requerido por los sistemas nacionales de educación.

Referente a la actividad docente en torno a las funciones logarítmicas, Farfán y Ferrari (2002) en su estudio reportan una enseñanza caracterizada por:
La ruptura que se percibe en la presentación escolar de los logaritmos, ésta es, como facilitadores de operaciones en un primer acercamiento de corte numérico y su posterior abordaje con todo rigor de su tratamiento como función sin que medie entre ambos la construcción de los mismos(...)

El abordaje de la funcionalidad de los logaritmos raya en lo axiomático, ya que a nuestro entender no existen elementos en el discurso escolar que propicien el pasaje de lo aritmético a lo analítico en el tratamiento de este concepto. (pp. 62-63)

Puntualizando un poco más, al considerar la interacción con los docentes de matemática de tales instituciones y el contenido propuesto en los textos de matemática, es razonable afirmar que la enseñanza de las funciones logarítmica y exponencial se evidencia un tratamiento axiomático a través de la exposición del profesor, ausencia de relaciones concretas con otras áreas del conocimiento matemático, tratamiento de la función logaritmo y exponencial en forma aislada con respecto a su génesis histórica, ausencia de modelizaciones de situaciones intra o extra matemática como lo es el problema del crecimiento, interés compuesto, magnitud de un sismo, entre otros.

El análisis precedente, podría relacionarse con la existencia de dificultades manifestadas por estudiantes de este nivel, durante el estudio $\mathrm{y}$ aprendizaje de la funciones logarítmica y exponencial (expresar definición, calcular valores de las variables $\mathrm{x}$ e $\mathrm{y}$ de las funciones para la construcción de la tabla de valores, representar gráficamente y analizar las funciones indicadas, distinguir propiedades logarítmicas, entre otros.)

Asimismo se puede hacer referencia la investigación que desarrolló Pacheco (2013) en titulada Organizaciones Matemática y Didácticas de las Practicantes Docentes. Caso Ecuación de 2do Grado con una incógnita cuyo propósito fue 
reconstruir las Organizaciones Matemáticas y las Organizaciones Didácticas de las ecuaciones de segundo grado con una incógnita desde las prácticas profesionales de la mención Matemática en la Facultad de Ciencias de la Educación de la Universidad de Carabobo. Para ello adoptó, un enfoque sistémico enmarcado dentro del paradigma interpretativo, empleando así la investigación de campo y documental; la cual además, se orienta por el método etnográfico y la teoría fundamentada. Llegando a la conclusión, la reconstrucción de las OM para las OD desde la praxis docente de las practicantes, muestra que a pesar de que intentan llevar a cabo un proceso de estudio conforme a la construcción de la OM: Ecuación de segundo grado con una incógnita y su OD correspondiente, no se logran gestar su construcción.

Además, las practicantes presentan la forma general de la ecuación de segundo grado como un elemento ostensivo y carente de justificación tecnológica-teórica; en otras palabras, no son presentados los elementos matemáticos que la conforman; tal situación conduce a una pérdida del significado estructural del objeto matemático, en contraposición con el esbozo de la Organización Matemática de Referencia (OMR). Por su parte, la OMR muestra cierta completitud, en las clases de las practicantes tiende a existir la incompletitud y la ausencia de los momentos didácticos, los cuales son fundamentales para la construcción y reconstrucción efectiva del proceso de estudio.

En virtud a lo expuesto, los autores citados en la descripción del problema planteado sostienen, sobre la base de los resultados de sus investigaciones, la preeminencia de elementos, inherentes a la problemática de la actividad docente del profesor de matemática, lo que trae consigo $u$ deterioro progresivo en la enseñanza de la matemática, disponibilidad de un conocimiento matemático incompleto, fragmentario, desarticulado y reduccionista; entre otros. Esto conduce, a los profesores, a cometer errores en la actividad matemática; propiciando así, el empleo de una enseñanza teórica de la matemática que limita la producción del conocimiento matemático de sus estudiantes.

En atención a lo expuesto, es pertinente interrogarse: ¿En qué consiste la actividad docente y la actividad matemática del profesor de matemática del cuarto (4to) año de la educación media general? En especial, en el municipio libertador ¿Cómo incide la actividad matemática en la actividad docente del profesor de matemática?; y en general, ¿Qué relaciones existen entre ambas actividades? Para abordar estas interrogantes en la presente investigación, se adopta el enfoque de la Teoría Antropológica de lo Didáctico (TAD), la cual situada en el marco del Programa Epistemológico de Investigación en Didáctica de las Matemáticas, postulada actividad matemática institucional como objeto primario de investigación.

Para ello, se emplea las nociones de Organización Matemática (OM), para referirse a lo matemático como resultado y Organización Didáctica (OD),también para el proceso de estudio o actividad de construcción y/o reconstrucción de lo matemático. En este sentido, la actividad matemática se integra en la noción de $\mathrm{OM}$ y la actividad docente en la noción de OD. En consecuencia, al considerar este modelo en didáctica de la matemática, se precisa reformular las interrogantes precedentes, empleando las nociones teóricas del mismo: ¿Qué relación existe entre la Organización Matemática y Organización Didáctica del profesor de matemática en torno a las funciones logarítmica y exponencial, en el cuarto (4to) año de Educación Media General del municipio libertador del estado Carabobo?

En función a lo anteriormente señalado, se estableció como objetivo general analizar la relación entre la Organización Matemática y la Organización Didáctica del docente de matemática en torno a las funciones logarítmica 
y exponencial, en el nivel del cuarto (4to) año de Educación Media General en el municipio Libertador del estado Carabobo.

Para ello en primer lugar se fijó establecer una aproximación a una Organización Matemática de Referencia en torno a las funciones logarítmica y exponencial; para luego describir las características de la misma y así Interpretar las relaciones entre las características de la Organización Matemática y la Organización Didáctica, puestas en juego, por el profesor de matemática durante el ejercicio de su actividad. Vale la pena hacer mención de las razones que justifican la realización de la presente investigación; entre ellas, su relevancia porque estudia la actividad matemática y la actividad docente del profesor de matemática, las cuales permiten comprender la relación entre lo matemático y lo didáctico; actividades éstas, que por ser un producto cultural, frecuentemente, no es cuestionada.

Tal situación además, ayuda a sugerir mejoras en los procesos de estudio llevados a cabo en la enseñanza de la matemática. De igual modo, es importante por cuanto la investigación se hizo en el marco de la Teoría Antropológico de lo Didáctico (TAD) para así obtener una interpretación a través de un modelo didáctico, que no es usual encontrar en los marcos teóricos de Venezuela.

Ahora bien, el estudio da su contribución a la línea de investigación enseñanza, aprendizaje y evaluación de la educación en matemática y educación en física; al permitir, partiendo de la información recabada, el mejoramiento de la praxis educativa en la enseñanza de la matemática. Al mismo tiempo, al enmarcarse en el modelo teórico: TAD, proporciona herramientas útiles que enriquecen a la línea de investigación, que sustenta el modelo teórico referido, y la cual se titulada: "La matemática como fuente esencial de proposiciones didácticas en la formación del profesor de matemática", no sólo en el nivel medio, sino también en el nivel superior, respecto al desarrollo de procesos de estudio en torno a las funciones trascendentales: función logarítmica y función exponencial.

Por otro lado, se subraya el interés de realizar un estudio enmarcado en el paradigma interpretativo y ubicado dentro del enfoque cualitativo en correspondencia con un método etnográfico; esto, ya que se buscó adentrarse en la realidad social y así observar lo ocurrido en el aula de clase, en relación a la enseñanza de matemática en el nivel medio. A partir de este punto, se destaca que la investigación es importante en lo académico e institucional, porque al constituirse el profesor como representante de una institución docente, siendo influenciado de una forma positiva por ésta; por consiguiente, la institución, la cual comprende el diseño currículo, normativas, pensum, entre otros, también, al disminuir la problemática de la enseñanza de la matemática es influenciada satisfactoriamente por las actividades que el docente realiza para encarar el proceso de estudio de una determinada OM.

\section{MÉTODO}

La metodología de esta investigación, se constituyó como el conjunto de condiciones que permitieron direccionar el estudio; esto, a objeto de dar respuesta satisfactoria a la situación problema planteada en una fase inicial del mismo. Subrayando, entre otros aspectos relevantes, la naturaleza del estudio, su paradigma, método, y estrategias empleadas. De ahí que, durante la fase actual, se hizo énfasis en el cómo se preparó tanto la recolección como el tratamiento, análisis y presentación de la información recabada.

El estudio se enmarcó dentro del paradigma interpretativo, el cual según lo planteado por Torres (citado en Goetz y LeCompte, 1988) se ocupa de indagar cómo los distintos actores humanos construyen y reconstruyen la realidad social mediante la interacción con los restantes 
miembros de su comunidad y para ello será necesario tener en cuenta la interpretación que ellos mismos realizan de los porque y para que de sus acciones y de la situación en general.

Los seres humanos según esta perspectiva, crean significados de su entorno social y físico, por tanto, de los comportamientos e interacciones de las personas y objetos en ese medio ambiente. Nuestras acciones están condicionadas por los significados que otorgamos a las acciones de las personas y a los objetos con los que nos relacionamos. Las investigaciones etnográficas son una de las alternativas que recogen esta filosofía interpretativa y reconstructiva de la realidad. (p. 13)

En acuerdo a lo expresado se abordó el problema de la actividad matemática y la actividad docente del profesor de matemática, en términos de su Organización Matemática (OM) y Organización Didáctica (OD) en el cuarto año de educación media en torno a las funciones logarítmica y exponencial, empleando para ello, procedimientos para recabar la información en el contexto ambiente donde se desenvuelven, y con el propósito de dar tratamiento a la información con objetividad, realizar su interpretación, describir la OM y OD, a fin de establecer las relaciones entre éstas.

Vale destacar que la organización didáctica está definida "por tareas y técnicas didácticas (componente técnico-práctico) así como una tecnología y teoría didáctica (componente discursivo) en las cuales se requiere la cooperación, contribución y participación tanto del alumno como el profesor" (Bosch, Espinoza y Gascón, 2003, p. 07). Mientras una organización matemática o noción de una praxeología:

Comprende la concepción del trabajo matemático como estudio de tipos de problemas o tareas problemáticas, así como caracterizar, delimitar y hasta clasificar los problemas en tipos de problemas; aunado, al entender, describir y caracterizar las técnicas empleadas para resolverlos, en base a argumentos sólidos que sustenten dichos técnicas. En otras palabras, permite la descripción del conocimiento matemático, en correspondencia, con el trabajo que involucra los elementos praxeológicos tipos de tareas, técnicas, tecnología y teoría. (Bosch, Espinoza y Gascón, 2003, p.05)

El estudio se enmarcó dentro de un enfoque cualitativo, en correspondencia a lo expresado, se abordó el problema de la OM y OD del profesor de matemática en torno a las funciones logarítmica y exponencial; en el cuarto (4to) año de educación media general, en virtud a las cualidades identificadas, en la estructura y dinámica de la realidad emergente de la interacción dentro de su contexto educativo y social. A tales efectos, "la investigación cualitativa se ubica y estudia lo fenómenos en su contexto estructural y situacional" (Martins y Stracuzzi, 2010). El método de la investigación se corresponde con una investigación etnográfica enmarcada dentro del paradigma interpretativo. Al respecto, Goetz y LeCompte (1988) sostienen:

Una etnografía es una descripción o
reconstrucción analítica de
escenarios y grupos culturales
intactos. Recrean para el lector, las
creencias compartidas, prácticas,
artefactos, conocimiento popular y
comportamientos de un grupo de
personas" Además, la etnografía es
un proceso, una forma de estudiar la
vida humana. (p. 28)

Asimismo, los autores antes mencionados Goetz y Lecompte (1988) subrayaron "los etnógrafos acostumbran a estudiar tal como ocurren naturalmente, en lugar de manipularlos o disponerlos anticipadamente bajo condiciones controladas"; de igual modo, refieren "El escenario natural, facilita el análisis sobre el terreno de causas y procesos, excluyendo al 
mismo tiempo un control estricto de las denominadas variables extrañas". (p. 35)

Es de hacer notar que esta investigación también fue de corte documental debido a la revisión y el análisis de contenido efectuado, ya que se recurrió a diversas fuentes para analizar interpretar la estructura de las funciones indicadas; propuesta tanto en libros de textos de matemática del cuarto año de educación media, para describir su evolución histórica.

Esto, en virtud a que el estudio requirió elaborar y definir una organización Matemática de Referencia (OMR): Primera actividad a realizar para estudiar las organizaciones matemáticas y didácticas del profesor y en consecuencia, dar cumplimiento al primer objetivo específico de la investigación. Además, el estudio es de caso, dado que se observa la enseñanza de dos (2) profesores en su actividad de aula.

De hecho, "El escenario natural, facilita el análisis sobre el terreno de causas y procesos, excluyendo al mismo tiempo un control estricto de las denominadas variables extrañas" (Goetz y Lecompte, 1988; p.35). Mientras que la investigación documental "se concreta exclusivamente en la recopilación de información en diversas fuentes" (p. 90)

En lo tocante al nivel, se hace referencia que el mismo se ubicó en un nivel exploratorio, en virtud a que se busca información relativa a la actividad matemática: OM y actividad didáctica: OD del profesor de matemática para familiarizar a la investigadora con las mismas, describir su OM y OD; y por consiguiente, interpretar las relaciones entre ambas. Tal situación, permite a la investigadora acercarse a aquellos fenómenos relativamente desconocidos y vinculados al tema estudiado; obtener así, información completa de un contexto específico de la vida real e investigar problemas del comportamiento profesor de matemática en su enseñanza de las funciones logarítmica y exponencial, considerados cruciales por profesionales de determinada área. En este orden de ideas, por un lado, Martins y Stracuzzi (2010) sostienen:
Es el inicio de cualquier proceso científico. Se realiza especialmente cuando el tema elegido ha sido poco examinado, es decir cuando no hay suficientes estudios previos y es difícil formular hipótesis. Se aplica cuando el tópico ha sido tratado escasamente, cuando no existe suficiente información o cuando no se dispone de medios para lograr mayor profundidad. (p. 92)

En cuanto a los informantes clave, estos fueron los profesores de matemática adscritos al Municipio Libertador Nro. 06, Parroquia Independencia, Valencia-Edo. Carabobo; que laboran tanto en las instituciones públicas como privadas; tales como U. E. Barrera y Crispín Pérez, respectivamente. Los mismos se estudiaron una vez iniciada la enseñanza del contenido hasta el día de la evaluación del mismo. Aunado, se consideraron a doce (12) estudiantes pertenecientes al grupo de alumnos, a cargo de los profesores ya mencionados, a los que se les analizaron los registros tanto de cada clase como de la evaluación desarrollada en torno a las funciones logarítmica y exponencial.

Como técnicas e instrumentos, se emplearon para el registro de la información que permitió analizar el estudio real y vivencial en el aula, la grabación de video y audio, así como las notas de campo para el caso de la observación participante, entrevista. Mientras que para el análisis del contenido sólo se utilizó un registro de campo descriptivo.

Los materiales a utilizar para registrar información durante la enseñanza de las funciones antes señaladas, comprenden: la grabadora de video y audio, un cuaderno de notas para el registro de campo. Aunado, a los registros de papel proporcionado en cuanto a los apuntes de clase de 12 estudiantes y sus evaluaciones. Incluso, las tablas de categorización, mencionadas previamente, plasmadas en formato de papel de carta, permitieron tabular, analizar e interpretar la información requerida en el estudio actual. 
Es importante destacar que para resguardar su identidad, se consideró llamar profesor $1\left(\mathrm{P}_{1}\right)$ y profesor $2\left(\mathrm{P}_{2}\right)$ y así como cuidar la objetividad propi la investigación científica. Cada una de ellas, estuvo a cargo de un curso de forma particular. $\mathrm{P}_{1}$ estuvo con el cuarto año sección "B" y $\mathrm{P}_{2}$ estuvo con el cuarto sección "B"; nivel perteneciente a la educación diversificada y profesional, de acuerdo con la Ley Orgánica de Educación (2007).

De igual modo, señaló que las clases siempre se realizaban con el grupo en aulas ya asignadas. En este sentido, $\mathrm{P}_{1}$ desarrolló dos (2) clases con el contenido de la función exponencial, centrándose solo en la representación gráfica de la función; en la primera clase asistieron 26, en la segunda 28 y en la tercera 27. Por su parte, $\mathrm{P}_{2}$ impartió dos clases de la función exponencial, en las que asistieron 26 y 27 estudiantes respectivamente, adicional, tres clases sobre la función logarítmica, haciendo énfasis en escribir expresiones de la forma logarítmica a la forma exponencial y viceversa; seguido, la representación gráfica $\mathrm{y}$ las propiedades logarítmicas. En estas sesiones de clases $\mathrm{P}_{2}$ contó con la asistencia de 26, 28 y 25 estudiantes respectivamente.

A esta altura, debo referir que hice registros mediante una grabadora de video (parcialmente) y una grabadora de audio, previo el consentimiento del directivo de las instituciones o campos de investigación. Naturalmente, se grabaron dos clases con grabadora de video y audio, y las restantes (una clase de $\mathrm{P}_{1}$ y tres clases de $\mathrm{P}_{2}$ ) fueron grabadas con grabadora de audio; esto, dada la ausencia del recurso audiovisual.

También, se tomaron algunas fotografías cuidando la identidad de los sujetos informantes, realicé notas de campo y solicité a las docentes su planificación, seis cuadernos y seis evaluaciones de sus estudiantes. Cabe señalar que las clases registradas fueron segmentadas en diversos episodios los cuales comprenden categorías específicas y descritas a continuación; categorías emergentes a partir de la Teoría Antropológica de lo Didáctico (TAD), propia de la presente investigación, a partir del lenguaje empleado por teóricos de la TAD; adaptándose en este sentido, a los objetivos propuestos inicialmente por este estudio.

\section{RESULTADOS}

En este apartado se presenta en primer lugar el trabajo documental con la finalidad de elaborar una aproximación a una Organización Matemática de Referencia (OMR) en torno a las funciones logarítmica y exponencial, en correspondencia con lo planteado en el primer objetivo de la investigación. Para ello, se realizó el análisis de contenido de los textos de matemática de nivel medio donde estas nociones son tratadas con fines instruccionales; esto servirá como referencia para analizar la actividad matemática y la actividad didáctica del profesor de matemática del nivel medio, en torno a las funciones logarítmica y exponencial.

Es importante destacar, se revisaron dos textos de matemática de cuarto año más utilizados, en cuanto a función exponencial como la función logarítmica, las cuales son caracterizadas a partir de la definición y las gráficas correspondientes. Donde se evidenció que se inició con tareas relativas a la definición y sus significados formales, representación gráfica de casos particulares y enunciación de propiedades en forma general; para luego, pasar a tareas específicas en las que las técnicas se derivan de las propiedades.

El lector se encuentra desde el comienzo con la tecnología y teoría; de tal forma que, en el primer encuentro convergen tres momentos didácticos; esto es, momento del primer encuentro (MPE), momento tecnológico-teórico (MTT) y momento de la institucionalización (MI). Tal situación, significa que el lector no tiene oportunidad alguna de explorar y construir las técnicas o propiedades. 
En cuanto a la relación entre la función exponencial y la función logarítmica, se inició con la función exponencial a partir de una $\mathrm{OM}$ previa: potenciación, y usando, la noción de función inversa se intenta introducir el logaritmo; y seguidamente, la noción de función logarítmica. Cabe destacar a continuación, algunas tareas generales evidenciadas en los textos analizados en este nivel medio, se presentan en el Cuadro 1:

Cuadro 1. Organización Matemática presente en los textos del nivel medio

\begin{tabular}{lll}
\hline $\begin{array}{l}\text { Tipos de Tareas y/o Tipos de } \\
\text { Problemas Matemáticos }\end{array}$ & \multicolumn{1}{c}{ Técnicas Matemáticas } & \multicolumn{1}{c}{ Tecnología-Teoría } \\
\hline $\begin{array}{l}\text { T1:Representación gráfica } \\
\text { de la función exponencial } \\
\text { de base } a .\end{array}$ & $\begin{array}{l}\mathrm{t}_{1} \text { : Construcción de tabla de } \\
\text { valores y Grafico de la función } \\
\text { exponencial. }\end{array}$ & $\begin{array}{l}\Theta_{1} \text { : Gráfico de la función } \\
\text { exponencial. }\end{array}$ \\
$\begin{array}{l}\text { T2:Representación gráfica } \\
\text { de la función exponencial } \\
\text { de base } a .\end{array}$ & $\begin{array}{l}\mathrm{t}_{2} \text { : Construir tabla de valores y } \\
\text { Grafico de la función }\end{array}$ & $\begin{array}{l}\Theta_{2} \text { : Propiedades de la función } \\
\text { logarítmica, Grafico de la } \\
\text { función logarítmica. }\end{array}$ \\
$\begin{array}{l}\text { T3: Calcular logaritmo de } \\
\text { base } a .\end{array}$ & $\begin{array}{l}\mathrm{t}_{3:} \text { Equivalencia entre } \\
\text { expresiones logarítmicas y } \\
\text { exponencial. }\end{array}$ & $\begin{array}{l}\Theta_{3} \text { : Concepto de logaritmo, } \\
\text { logaritmos } \\
\text { antilogaritmos. }\end{array}$ \\
\hline
\end{tabular}

Ahora bien, en cuanto a la observación de cada uno de los episodios ejecutados por cada una de las docentes, se presenta la Cuadro 2, con algunos componentes clave desarrollados en cuanto a los tipos de tareas y la técnica y tecnología asumida. Se puede interpretar según lo observado, en primer lugar que las tareas matemáticas planteadas fueron centradas en graficar función exponencial, en la $\mathrm{OM}$ de $\mathrm{P}_{1} \mathrm{y}$ graficar función exponencial y logarítmica de base a, hallar equivalencias entre expresiones de la forma logarítmica y la forma exponencial, despejar variable $\mathrm{x} / \mathrm{y}$, hallar inversa de una función, introducir logaritmos, definir logaritmos, definir logaritmos especiales y resolver logaritmos aplicando propiedades de la función logarítmica, en la $\mathrm{OM}$ de $\mathrm{P}_{2}$.

Dejando a un lado, ambas profesoras, las tareas matemáticas que propicien el cuestionamiento tecnológico y la justificación de las tareas planteadas, así como interpretación del bloque tecnológico-teórico. Tareas que implicaron establecer la relación entre ambas funciones, realizar cálculos logarítmicos $\mathrm{y}$ exponenciales, interpretar los resultados obtenidos y modelizar situaciones de la vida real mediante las funciones logarítmica $\mathrm{y}$ exponencial, entre otras relevantes; por lo cual se evidencia una desarticulación en su praxeología, la cual "se deriva de la unión de los términos praxis que significa saber-hacer y logos que significa saber-razón" (Bosch, Espinoza y Gascón, 2003, p. 05).

Aunado a esto, se puede advertir en lo concerniente a la organización matemática enseñada efectivamente por las profesoras del nivel de cuarto año en torno a las funciones logarítmica y exponencial, no se corresponde con la OM de referencia la cual es relativamente completa, no sólo en cuanto al nivel de completitud de los bloques prácticos y discursivos; sino también, porque para su elaboración se tomaron en consideración, entre otras tareas y elementos, situaciones problema de naturaleza matemática y extra matemática que, de alguna forma, permiten dar cuenta tanto de su razón de ser como de los objetos matemáticos que comprende. 
Cuadro 2. Organización Matemática de Referencia

Tipos de Tareas y/o Tipos de Problemas Matemáticos

Problema Generador o Razón de ser de la $\underline{\text { OMR }}$

I. Dado un número real llamado base a y $\mathbf{x}$ llamado exponente: ¿Cuál es el resultado de calcular $a^{x}$ ? ¿Cuáles son las características de esa operación?

II. Dado y y a ¿Cuál es el exponente al cual hay que elevar la base y?

III. ¿Qué relación es posible establecer entre estos problemas I y II?

La respuesta al problema planteado, se corresponde con los Tipos de Tareas siguientes:

T1: Realizar operaciones con potencias en R.

T2: Representación gráfica de la función exponencial (natural y de base a).

T3: Representación gráfica de la función logarítmica (natural y de base a).

T4: Relacionar la función exponencial con la función logarítmica.

T5: Aplicar las propiedades logarítmicas.

T6: Resolver ecuaciones logarítmicas y exponenciales.

T7: Realizar cálculos a través de los logaritmos y las exponenciales.

T8: Modelizar situaciones de la vida real a partir de la función logarítmica y exponencial.

T9: Interpretar resultados al emplear la función logarítmica y exponencial

T10: Justificar técnicas de la función logarítmica y exponencial.

T11: Describir tareas y técnicas inversas de las funciones logarítmica y exponencial.

T12: Construir situaciones problemas que puedan ser modelizadas a través de las funciones logarítmicas y exponencial.
Técnicas Matemáticas
A) Técnicas relativas al cálculo de potencias

1) Técnica basada en el desarrollo de potencias

2) Técnica basada en el desarrollo de operaciones básicas en $\mathrm{R}$.

B) Técnicas relativas a la representación y/o estudio de la gráfica de funciones exponencial y logarítmica

1) Técnica basada en la aplicación de la definición de la función exponencial

2) Construcción de tabla de valores

3) Análisis e interpretación una expresión de la forma exponencial

4) Equivalencia entre expresiones de la forma logarítmica y la forma exponencial

5) Análisis gráfico.

6) Progresiones aritméticas y geométricas

C) Técnicas relativas a la aplicación de las propiedades logarítmicas

1) Técnica basada en el desarrollo de logaritmo a través de propiedades.

D) Técnicas relativas a la resolución de ecuaciones logarítmicas y exponencial

1) Técnica basada en la transformación a ecuaciones lineales.

2) Técnica basada en la aplicación de logaritmos.

\section{E)Técnicas relativas al cálculo con logaritmos y} las exponenciales

1) Técnica basada en el desarrollo de potencias

2) Técnica basada en la equivalencia entre expresiones de la forma logarítmica y la forma exponencial

3) Técnica basada en el desarrollo de logaritmo a través de propiedades logarítmicas.

4) Técnica basada en el cálculo de logaritmos y antilogaritmos a través del uso de la calculadora.

\section{CONCLUSIONES}

Una vez interpretada la información obtenida del trabajo de campo, se puede decir, se logró analizar la relación entre la Organización Matemática y la Organización Didáctica del docente de matemática en torno a las funciones logarítmica y exponencial, en el nivel del cuarto año de Educación Media General en el municipio Libertador; por tanto se encontró en la organización didáctica las siguientes características: hace énfasis en el momento del primer encuentro, centrado en la presentación del componente tecnológico-teórico. 
El encuentro de los estudiantes con la OM en estudio o con algunos tipos de tareas correspondiente tiene lugar, en todos los casos, en convergencia con un parcial momento del trabajo de la técnica y el momento de la institucionalización, en detrimento del momento exploratorio y el momento tecnológico-teórico. Dicho de otro modo, los estudiantes se encuentran directamente con los elementos tecnológicos-teóricos de la OM ya institucionalizada, a través de la presentación directa, realizada por el profesor. Así pues, no se evidencia el encuentro con un tipo de problema o tarea que dé lugar a la elaboración; aun cuando insuficiente, de una técnica, de su práctica y de la posibilidad de construcción, al menos de algunos de los elementos praxeológicos.

Los docentes informante clave presentaron el tema dictando, entre otros elementos, la definición de las funciones logarítmica $y$ exponencial, y plantean sus ejemplos respectivos. Las actividades llevadas a cabo en clase tanto por los estudiantes como, esencialmente, por las profesoras son actividades para presentar respuestas matemáticas sin interrogantes que den lugar a tales respuestas. Cuando las profesoras presentan las definiciones $\mathrm{y}$ propiedades relativas a la función exponencial y a la función logarítmica, lo que hacen es presentar las respuestas a un conjunto de problemas acumulados en la cultura matemática a lo largo de la historia; sin tomar en cuenta que las respuestas son tales en tanto exista alguna pregunta, interrogante o problema a partir de los cuales se elabora. En síntesis, finalmente se puede decir la actividad docente y la actividad matemática del profesor de matemática del cuarto (4to) año de la educación media general especificadas anteriormente, revelaron la visión que la institución docente; y en consecuencia las profesoras, tienen de la matemática y en particular, de la función exponencial de la función logarítmica; al interpretar estos objetos matemáticos como obras ya acabadas, sin posibilidad de reconstrucción.

\section{REFERENCIAS}

Báez, M., Cantú, C. y Gómez, K. (2007). Un Estudio Cualitativo Sobre las Prácticas Docentes en el Aula de Matemáticas en el Nivel Medio. (Tesis de Grado). Universidad Autónoma de Yucatán. México. Recuperado de http://www.uady.mx/ matemati/dme/docs /tesis/TesisGrupal_Baez-Cantu-Gomez.pdf

Banco Interamericano de Desarrollo (2010). La Condición de la Educación Matemáticas y Ciencias Naturales en América Latina y el Caribe (ALC). Recuperado:https://publications.iadb.org/pu blications/spanish/document/Lacondici\%C3\%B3n-de-la-educaci\%C3\%B3n-enmatem\%C3\%A1ticas-y-ciencias-naturales-enAm\%C3\%A9rica-Latina-y-el-Caribe.pdf

Bastán, M., Buffarini, F., Lisera, M. y Roso, F. (2006). Problemática del Profesor de Matemática Atendiendo a los Niveles de determinación de lo Didáctico. I REPEM - Memorias. Universidad Nacional de Río Cuarto - Argentina

Bosch, M., Chevallard, Y. y Gascón, J. (1997). Estudiar matemáticas. El Eslabón Perdido Entre Enseñanza y Aprendizaje. Barcelona: ICEHorsori

Bosch, M., Fonseca, C. y Gascón, J. (2000). Incompletitud de las organizaciones matemáticas locales en las instituciones escolares. Recuperado de

https://books.google.co.ve/books?id=inpcdw aaqbaj\&pg=pa116\&lpg=pa116\&dq=bosch, $+\mathrm{m}$., + fonseca, $+\mathrm{c} .+\mathrm{y}+\mathrm{gasc} \% \mathrm{c} 3 \% \mathrm{~b} 3 \mathrm{n},+\mathrm{j} .+(2000)$.+inco mpletitud+de+las+organizaciones+matem $\% c 3$ \%a1ticas+locales+en+las+instituciones+escolar es\&source=bl\&ots=tpb9oxzso4\&sig=acfu3u0p 27ybsoowejaxwjamlsp2lk_ccw\&hl=es419\&sa= x\&ved=2ahukewi1jldyipbpahwkut8kheogbc8 q6aewanoecaoqaq

Bosch, M. (2003). Un punto de vista antropológico: la evolución de los instrumentos de representación en la actividad matemática. Universidad Ramón Llull

Bosch, M., Espinoza, L. y Gascón, J. (2003). El profesor como director de estudio. Análisis de organizaciones didácticas espontáneas. Recherhes en Didáctique de Mathemátiques. 23(1), pp.79-135

Farfán, R. y Ferrari, M. (2002). Una visión socioepistemológica. Estudio de la función logaritmo. Acta Latinoamericana de Matemática Educativa (RELIME).15 (2) Recuperado: http://www.clame.org.mx/documentos/alm e15_2.pdf [Consulta: 20 Noviembre, 2010] 
Goetz, J. y Lecompte, M. (1988). Etnografía y diseño cualitativo en investigación educativa. Madrid: Morata

Ley Orgánica de Educación (2009). Gaceta Oficial de la República Bolivariana de Venezuela No. 5.929, agosto 15,2009

Organización de las Naciones Unidas para la Educación, la Ciencia y la Cultura, instituto Internacional para la Educación Superior. (2010). Informe de gestión 2008-2010. Recuperado:

https://www.un.org/ruleoflaw/es/un-andthe-rule-of-law/united-nations-educationalscientific-and-cultural-organization/
Pacheco, V. (2013). Organizaciones Matemáticas y Didácticas de los Practicantes-Docentes. Caso Ecuación de 2do Grado con una Incógnita. (Tesis de Maestría). Universidad de Carabobo. Recuperado de http://produccionuc.bc.uc.edu.ve/documentos/trabajos/70003 62D.pdf

Szemruch, V. y Vaccaro, D. (2002). Logaritmos, Física y Algo Más. Acta Latinoamericana de Matemática (RELIME).

11 Recuperado:http://www.matedu.cicata.ipn.m x/documentos/alme/alme11.pdf 